\title{
OPTICAL AND ELECTRICAL STUDIES OF FR1 AND FR2 DEFECTS IN GaAs*
}

\author{
R. DWILIŃSKi ${ }^{a}$, M. PALCZEWSKA ${ }^{b}, \mathrm{P} . \mathrm{KACZOR}^{c}, \mathrm{~K} . \mathrm{KORONA}^{a}$, \\ A. WYSMOLEK ${ }^{a}$, R. BożEK ${ }^{a}$ AND M. KAMIŃSKA ${ }^{a}$ \\ a Institute of Experimental Physics, Warsaw University \\ Hoża 69, 00-681 Warszawa, Poland \\ ${ }^{b}$ Institute of Electronic Materials Technology, Wólczyńska 133, 01-919 Warszawa, \\ Poland \\ cInstitute of Physics, Polish Academy of Sciences \\ Al. Lotników 32/46, 02-668 Warszawa, Poland
}

The systematic EPR, optical absorption, photoluminescence and thermally stimulated current studies of acceptor defects in bulk GaAs were performed. For the first time, parallel EPR and optical absorption experiments allowed to find the absorption spectrum due to the photoionization of FR1 defect with the threshold at $0.19 \mathrm{eV}$. Photoluminescence studies showed two families of bands in the energy range of about 1.25 to $1.35 \mathrm{eV}$. We tentatively ascribed them to FR1 and FR2 complexes with shallow donors. Thermally stimulated current measurements showed two peaks at $90 \mathrm{~K}$ and $110 \mathrm{~K}$ assigned to FR1 and FR2 respectively.

PACS numbers: 71.55.-i, 78.50.Ge, 76.30.Mi

\section{Introduction}

On the basis of existing experimental data it can be safely concluded that the compensation mechanism in commercially grown $\mathrm{GaAs}$ is not a simple balance between shallow donors, carbon acceptor and EL2 [1]. The concentration of carbon acceptor is not sufficient to explain the experimentally found concetration of ionized EL2. Therefore, quite a big interest has been concentrated on the origin of possible acceptor defects in GaAs for the last few years. Using EPR technique so-called FR1 and FR2 dcfect acceptors have bcen found in GaAs crystals [2]. However, their EPR spectra have not been resolved enough to determine the origin of these defects.

\footnotetext{
*This work is supported in part by the Committee for Scientific Research 201799101 grant.
} 


\section{Experiment}

Using EPR technique, GaAs crystals with high concentration of FR1 and FR2 defects were chosen. The observed features of these defects (EPR and foto-EPR spectra) were the same as published in literature [2,3]. Moreover, it was found that:

- both defects existed in crystals pulled from quartz crucibles beside grown by LEC (liquid encapsulated Czochralski) method;

- FR1 in Cr-doped $\left(10^{18} / \mathrm{cm}^{3}\right)$ crystals did not have metastable features [4].

This indicated that metastability of FR1 in undoped crystals is caused by holes created during the $A s_{\mathrm{Ga}}^{+}$metastable transition: $A \mathrm{~s}_{\mathrm{Ga}}^{+}+\mathrm{e}_{v}^{-} \longrightarrow A \mathrm{~s}_{\mathrm{Ga}}^{0} \longrightarrow A \mathrm{~s}_{\mathrm{Ga}}^{*}$ (metastable).

For the first time, parallel EPR and optical absorption experiments allowed to find the absorption spectrum due to the photoionization of FR1 defect with the threshold at $0.19 \mathrm{eV}$ (Fig. 1). The best fit to the experimental curve (shown in Fig. 1) was obtained by applying Lucovsky model [5] for photoionization of localized defects, completed by J. Langer by including electron-phonon coupling [6]. Parameters of this fit will be discussed later. The identification of this spectrum was further confirmed in Cr-doped GaAs, where FR1 behaviour under illumination was different than in undoped $\mathrm{GaAs}$ and did not show metastability after switching the white light off both in EPR and absorption experiments.

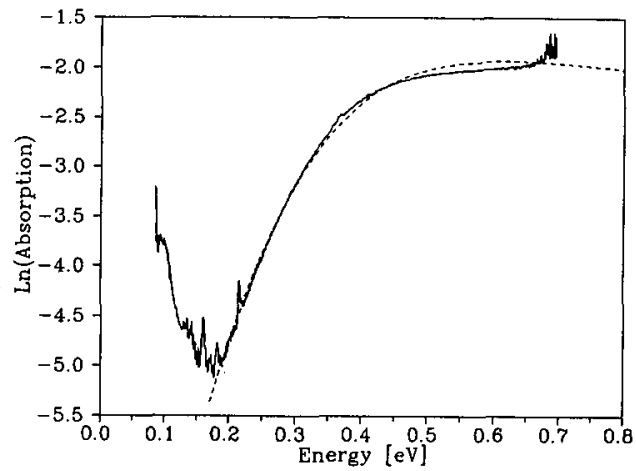

Fig. 1. Absorption spectrum due to the photoionization of FR1 defect. The fitted curve was obtained by applying Lucovsky-Langer model $[5,6]$.

Photoluminescence (PL) studies of samples with high concentration of FR1 showed a family of bands in the energy range of about $1.35 \mathrm{eV}$, which moves to $1.25 \mathrm{eV}$ with increasing relative concentration of FR2 (Fig. 2). The intensity of this luminescence was higher for slightly $n$-type crystals than for semi-insulating ones for adequate concentrations of FR defects. We tentatively ascribed this luminescence to FR1 and FR2 complexes with shallow donors.

Thermally stimulated current (TSC) measurements (Fig. 3) showed different peaks depending on illumination times and on wavelength of excitation light. The 


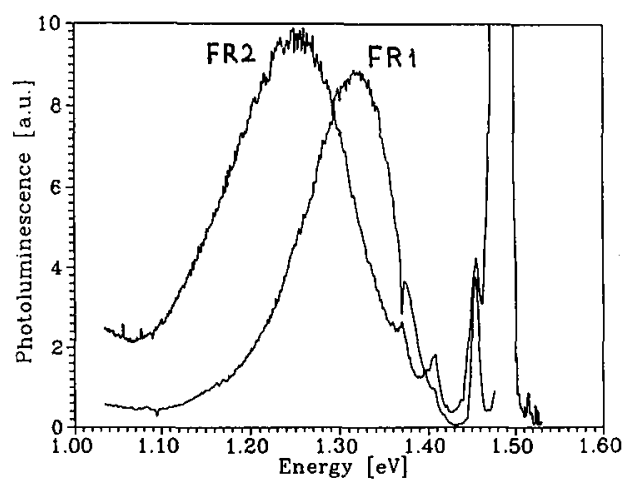

Fig. 2. PL bands for samples with high concentrations: of FR1 $(1.35 \mathrm{eV})$ and of FR2 $(1.25 \mathrm{eV})$.

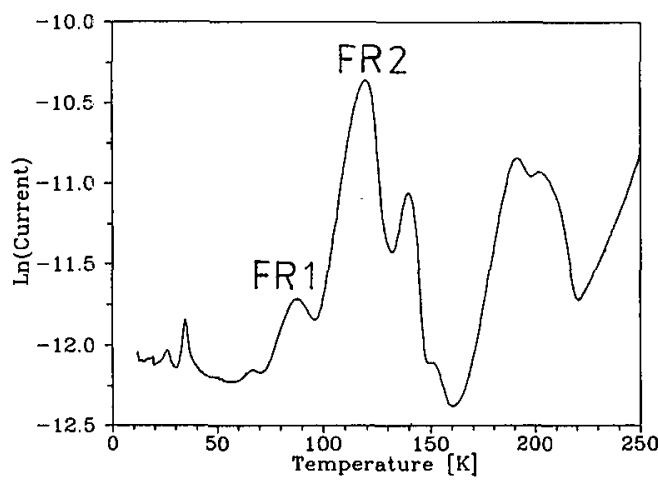

Fig. 3. TSC peaks at $90 \mathrm{~K}$ and $110 \mathrm{~K}$ assigned to FR1 and FR2, respectively.

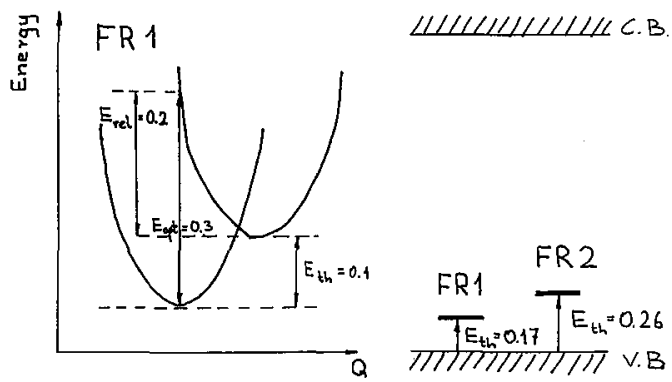

Fig. 4. Configuration diagram for FR1 and energy positions of FR1 and FR2 with GaAs band gap. Energy values were obtained from Lucovsky-Langer model, PL and TSC results. 
peak at $90 \mathrm{~K}$ was created by about 10 minutes of lighting whereas the peak at $110 \mathrm{~K}$ by 1 second of lighting. Similar differences were observed by EPR technique: EPR spectrum due to FR2 appeared after short (about $1 \mathrm{~s}$ ) illumination, whereas FR1 signal needed long (10 min) illumination to saturate. Furthermore, the peak at $90 \mathrm{~K}$ was most efficiently created by $1.2 \mathrm{eV}$ light and the peak at $110 \mathrm{~K}$ by $0.9 \mathrm{eV}$ light. Based on this, the peaks at $90 \mathrm{~K}$ and $110 \mathrm{~K}$ wcre assigned to FR1 and FR2, respectively.

\section{Conclusion}

As a result of the performed studies it was possible to settle the energy position of FR1 and FR2 defects within the energy band scheme of GaAs (Fig. 4).

For FR1 defect:

(a) the fit to the absorption data gave the value of thermal energy $E_{\text {th }}=0.10 \pm$ $0.05 \mathrm{eV}$, optical energy $E_{\text {opt }}=0.30 \pm 0.02 \mathrm{eV}$ and lattice relaxation cnergy $E_{\text {rel }}=0.20 \pm 0.05 \mathrm{eV}$;

(b) from the position of TSC peak at $90 \mathrm{~K}$ the thermal energy applying Bube's [7] formula: $E_{\text {peak }}=k T_{\mathrm{m}} \ln \left(T_{\mathrm{m}}^{4} / \beta\right)$ (where $T_{\mathrm{m}}$ means the temperature at the current peak's maximum and $\beta$ - the heating rate during the thermal scan) was obtained $E_{\mathrm{th}}=0.17 \pm 0.02 \mathrm{eV}$;

(c) the energy position of $1.35 \mathrm{eV}$ PL peak is consistent with FR1 energy obtained from absorption and TSC measurements.

For FR2 defect:

(a) from TSC measurements thermal energy $E_{\text {th }}=0.26 \mathrm{eV}$;

(b) PL results are consistent with TSC ones;

(c) comparison with Williams studies [8] confirms gallium vacancy related character of FR2.

\section{References}

[1] E.R. Weber, M. Kamińska, in: Proc. 5th Conf. Semi-Insulating III-V Materials, Malmö, (Sweden), 1988, Adam Hilger, Bristol 1988, p. 111.

[2] M. Baeumler, U. Kaufmann, J. Windscheif, W. Wilkenig, in: Proc. Conf. EMRS, Adv. Mater. Telecommunications, Strassbourg (France) 1988, Vol. XIII, Ed. P.A. Glasgow, p. 111.

[3] M. Hoinkins, E.R. Weber, in Ref. [1], p. 43.

[4] M. Palczewska, R. Jabłon'ski, Acta Phys. Pol. A77, 339 (1990).

[5] G. Lucovsky, Solid State Commun. 3, 229 (1965).

[6] U. Piekara, J.M. Langer, B. Krukowska-Fulde, Solid State Commun. 23, 583 (1977).

[7] R.H. Bube, Photoconductivity of Solids, Wiley, New York 1960.

[8] E. Williams, H.B. Bebb, in: Semiconductors and Semimetals, Vol. 8, Ed. R.K. Willardson, A.C. Beer, Academic Press, New York 1972, p. 321. 